\title{
PLANAR TRANSMISSION LINES IN MW AND MMW CIRCUITS
}

\author{
Ján Zehentner, Jan Macháč, Jan Mrkvica
}

\begin{abstract}
Absract - The analysis of open, particularly slotted planar transmission lines in a wide frequency range is presented in this paper. The full wave approach by the spectral domain method is applied. Theoretical findings and their practical consequences are reported. Some outputs from the CST Microwave Studio and from our Analysis of Planar Transmission Lines package are shown.
\end{abstract}

Keywords - Planar transmission lines, Spectral domain method, Dispersion characteristics, Bound waves, Leaky waves.

\section{INTRODUCTION}

Mankind knows two ways of transmitting information, signals or power from one place to another, either by propagation along a line connecting the two places, or by propagation in open air. The latter requires the signal to be transformed from a circuit into a wave radiated by a transmitting antenna. The wave then propagates towards the receiving antenna, where it is transformed back into a signal to be processed further in circuits. In this paper we are engaged in the first variant, i. e., transmission of information by a line.

A transmission line is a technical tool for transferring a signal along a path routed in space, customarily by an abrupt change of the medium parameters such as conductivity, permittivity or permeability. The signal propagates along surfaces on which at least one of the above mentioned parameters changes rapidly. In the case of an abrupt change of the conductivity we have a metallic line. Dielectric lines are based on a change of permittivity and, by analogy, there is a change of permeability. We will consider metallic and dielectric lines, the most applied types of lines.

From the chronological point of view, the development of different types of lines was influenced by the frequencies of the available generators and by the way that the lines were produced. At first, the classic coaxial lines were used in the decimetre wavelength range, utilizing the propagation of TEM waves. The theory of wave propagation on a coaxial line has been perfectly and completely elaborated. The analysis and design of this line is therefore relatively simple. Nowadays coaxial lines are used up to $70 \mathrm{GHz}$.

The next stage in line development involves the waveguides successfully utilized up to now in $\mathrm{cm}$ and $\mathrm{mm}$ technology. Here again, the theory is complete, and it enables

Ján Zehentner and Jan Macháč are with the Faculty of Electrical Engineering, Czech Technical University in Prague, Technická 2, 16627 Prague 6, Czech Republic, E-mail: zehent@,feld.cvut.cz, machac@,fel.cvut.cz.

Jan Mrkvica is now with RETIA, a. s., Pražská 341, 53002 Pardubice, Czech Republic, mrkvicj@,seznam.cz. us to investigate the waveguide behavior, to design waveguides accounting for different demands following from the functionality of a line within the circuit. In parallel, the theory of dielectric lines was built up. Useful features of dielectric lines were utilized in special cases of signal propagation, e. g., in microwave spectroscopy and in communication systems, particularly in the $\mathrm{mm}$-wave range. Space-reduced versions of dielectric waveguides are optical fibers and other light guides used in optical signal processing.

Advances in semiconductor technology, production of high quality organic materials or ceramics with a low loss factor, and the availability of low cost printed circuit boards opened up the latest stage in transmission line development. A planar arrangement, usually a combination of dielectrics and metallization, is typical for transmission lines. Nowadays there is a numerous group of lines differing from each other just by the arrangement of these two materials. At lower frequencies they are widely called printed circuit lines. At higher frequencies each line carries its own specific name.

Low losses, negligible signal distortion, high transmitted power capability, electromagnetic compatibility, a wide operational frequency band, high electromagnetic resistance and pure dominant mode propagation are requirements set on any kind of transmission line, along with easy and low cost production, and high integrability with various passive circuits and active devices. Transmission lines, such as waveguides or coaxial lines, transmit waves in a space totally confined by metallic walls, so they satisfy all mentioned demands. Compared with them, open transmission lines, where waves propagate along the line and its close vicinity, such as two wire lines, striplines, microstrip lines, image guides, uniplanar lines, etc., have reduced transmitted power, higher losses, lower electromagnetic resistance, cross-talk to neighboring circuits and occasionally strong dispersion. The features of closed and open lines overlap when a line belongs to the finlines group. However, they are not easy to produce.

Planar technology has been developed in the last fifty years and has enabled the fabrication of printed circuit lines. These were first investigated in the framework of the quasi-static approach. Only propagation of the dominant modes was accounted for, and resulted in the closed-form formulae of the propagation constant and characteristic impedance valid at low frequencies. Next, the dispersion of the dominant modes was described with limited validity by various dispersion models. Several useful books are available [1-5] from that period. Finally, the full wave approach dominated and provided a realistic picture of the propagation of the bound modes, and at higher frequencies also the leakage effects. The leakage could be into the substrate or into the space. It needs to be suppressed in circuits, since leakage deteriorates circuit operation, but on the other hand it should be supported in 
antenna applications. To provide an insight into the physics of wave propagation along the line, and leakage, use is made of theoretical analysis, visualization of transmitted modes and measurements. A change in the cross-sectional size of a line, the material parameters or bending from the straight direction results in many types of inhomogeneities, which are sources of undesired radiation, power leakage and propagation of higher modes. Generally available SW packages devoted to analysis and design of these lines mostly have not taken these effects into consideration. They provide solutions satisfactory only for purely bound modes at low frequencies. At higher frequencies, when leakage effects or higher order modes appear, the designer must either accept the limits of the code validity, or rely on the results without being able to check them. This factor in many cases leads to discrepancies between theoretically predicted and measured characteristics of circuits or systems containing active devices or passive elements.

In the following sections we first introduce the well-known method of moments applied in the spectral domain finalized by a root search on the complex plane of the spectral variable used for analysis of the lines. By means of the dispersion characteristics, we explain the behavior of the line depending on its cross-sectional proportions and substrate permittivity. We have elaborated a code for analyzing eighteen planar transmission lines calculating the propagation constants of particular modes and, when applicable, also the characteristic impedance. The code enables the frequency limits to be found when higher order bound or leaky modes set up. The CST Microwave Studio provides us with a practical idea about the total field propagation under specific conditions of excitation. The applicability of particular lines will be pointed out. We have checked some new findings by measurements on enlarged scale models of the lines. Excitation of planar lines by a real source is attracting the current interest of researchers. It turns out that, in the close vicinity of the source, standard transmission line theory no longer holds, due to the presence of so called residual modes. We have observed their presence in experiments.

\section{A LitTLE BIT TheORY Will Not Go Amiss}

The propagation constant, generally complex, and the field distribution represent individual modes propagating on a line. The characteristic impedance, if its definition has a physical meaning, supplements the picture of the mode. Full information about the mode is provided by the wave equation. Several methods have been developed for solving it [6-7]. However, it turned out that the spectral domain method has become the most popular and widely used technique for analyzing planar transmission lines. The original approach was published in two short papers, one dealing with a slotline [8] and the second treating a microstrip line [9]. Many works have successfully demonstrated the power, accuracy and numerical efficiency of this method. Some terms used in the literature dealing with field propagation over the line were derived from this method, so it will be helpful to recall the main steps. We will explain briefly the procedure, for instance, on the slotline, Fig. 1.

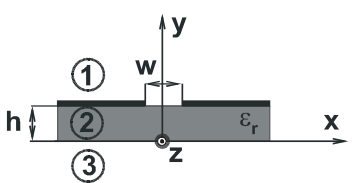

Fig. 1 Cross-section of a slotline.

Perfectly conductive strip conductors with infinitesimal thickness are assumed in the spectral domain method (SDM). We investigate the field represented by constituent modes in the source-less region, i. e., regardless of how they are excited. The concept of transversal waves is adopted, and let all the field components have a dependence of the form $e^{-j \gamma_{z} z}$ where the propagation constant in the $\mathrm{z}$ direction $\gamma_{z}=\beta-j \alpha, \beta$ is the phase constant, while $\alpha$ is the attenuation constant. The electric $\boldsymbol{\Phi}^{\mathbf{e}}=\mathbf{y}_{\mathbf{0}} \boldsymbol{\Phi}^{e}$ and magnetic $\boldsymbol{\Phi}^{\mathbf{m}}=\mathbf{y}_{\mathbf{0}} \boldsymbol{\Phi}^{m}$ Hertz potential determine the TM and TE waves, respectively. Hereafter the upper indices of the potentials will be dropped. The variable separation applied in the wave equation being solved for the field in Cartesian co-ordinates results in the equation

$$
\left\lfloor\Delta+\left(k_{j}^{2}-\gamma_{z}^{2}\right)\right\rfloor \Phi(x, y)=0
$$

where $k_{j}$ is the wave number in the corresponding medium, $\mathrm{j}=1,2,3$ in Fig. 1. Eq. (1) is a $2 \mathrm{D}$ task. To facilitate the solution, the dependence on the $x$ variable is removed by the Fourier transform

$$
\widetilde{\Phi}(\xi, y)=\int_{-\infty}^{\infty} \Phi(x, y) e^{-j \xi x} d x
$$

The backward Fourier transform provides the original potential

$$
\Phi(x, y)=\frac{1}{2 \pi} \int_{-\infty}^{\infty} \widetilde{\Phi}(\xi, y) e^{j \xi x} d \xi
$$

Applying Eq. (2) in Eq. (1) one gets

$$
\frac{\partial^{2} \widetilde{\Phi}(\xi, y)}{\partial y^{2}}-\gamma_{j}^{2} \widetilde{\Phi}(\xi, y)=0,
$$

where

$$
\gamma_{j}^{2}=\xi^{2}+\gamma_{z}^{2}-k_{j}^{2}
$$

The solution of Eq. (4) is known, and contains eight integration constants. Their interrelations determine the boundary conditions transformed also into the spectral domain on the plane $y=0$

$$
\begin{aligned}
& \widetilde{E}_{x 2}(\xi, 0)=\widetilde{E}_{x 3}(\xi, 0) \\
& \widetilde{E}_{z 2}(\xi, 0)=\widetilde{E}_{z 3}(\xi, 0)
\end{aligned}
$$

and on the plane $y=h$

$$
\widetilde{E}_{x 1}(\xi, h)=\widetilde{E}_{x 2}(\xi, h)
$$




$$
\begin{gathered}
\widetilde{E}_{z 1}(\xi, h)=\widetilde{E}_{z 2}(\xi, h) \\
\widetilde{H}_{x l}(\xi, h)-\widetilde{H}_{x 2}(\xi, h)=-\widetilde{J}_{z}(\xi, h) \\
\widetilde{H}_{z 1}(\xi, h)-\widetilde{H}_{z 2}(\xi, h)=\widetilde{J}_{x}(\xi, h)
\end{gathered}
$$

where $\widetilde{J}_{x}(\xi, h), \widetilde{J}_{z}(\xi, h)$ are current densities. The substitution of the integration constants into Eqs. (10) and (11) provides

$$
\begin{aligned}
& \widetilde{J}_{z}(\xi, h)=\widetilde{Y}_{z z}(\xi) \widetilde{E}_{z l}(\xi, h)+\widetilde{Y}_{z x}(\xi) \widetilde{E}_{x l}(\xi, h) \\
& \widetilde{J}_{x}(\xi, h)=\widetilde{Y}_{x z}(\xi) \widetilde{E}_{z l}(\xi, h)+\widetilde{Y}_{x x}(\xi) \widetilde{E}_{x l}(\xi, h)
\end{aligned}
$$

where $\widetilde{Y}_{m n}(\xi)$ are Green's functions in the spectral domain. We have two equations with four unknowns $\widetilde{J}_{z}, \widetilde{J}_{x}, \widetilde{E}_{z 1}, \widetilde{E}_{x 1}$. Let $\widetilde{E}_{z I}(\xi, h), \widetilde{E}_{x I}(\xi, h)$ be written in the form of the series of basis functions $\tilde{e}_{z n}(\xi), \tilde{e}_{x n}(\xi)$

$$
\begin{aligned}
& \widetilde{E}_{z l}(\xi, h)=\widetilde{e}_{z}(\xi, h)=\sum_{n=1}^{\infty} c_{z n} \widetilde{e}_{z n}(\xi) \\
& \widetilde{E}_{x l}(\xi, h)=\widetilde{e}_{x}(\xi, h)=\sum_{n=1}^{\infty} c_{x n} \widetilde{e}_{x n}(\xi)
\end{aligned}
$$

where $c_{z n}, c_{x n}$ are unknown amplitudes of these functions. Now Galerkin's method, i. e., the method of moments in the spectral domain, and Parseval's theorem are applied in Eqs. (12) and (13). The current densities and electric field components are nonzero in the complementary regions of $x$, $y=h$. Consequently, $\widetilde{J}_{z}(\xi), \widetilde{J}_{x}(\xi)$ vanish from Eqs. (12) and (13), and a homogeneous set of algebraic equations remains in terms of amplitudes $c_{z n}, c_{x n}$

$$
\begin{aligned}
& \sum_{m=1}^{\infty}\left[c_{z m} K_{z z}+c_{x m} K_{z x}\right]=0 \\
& \sum_{m=1}^{\infty}\left[c_{z m} K_{x z}+c_{x m} K_{x x}\right]=0
\end{aligned}
$$

where

$$
\begin{aligned}
& K_{z z}=\int_{-\infty}^{\infty} \widetilde{Y}_{z z}(\xi) \widetilde{e}_{z m}(\xi) \widetilde{e}_{z n}^{*}(\xi) d \xi \\
& K_{z x}=\int_{-\infty}^{\infty} \widetilde{Y}_{z x}(\xi) \widetilde{e}_{x m}(\xi) \widetilde{e}_{z n}^{*}(\xi) d \xi \\
& K_{x z}=\int_{-\infty}^{\infty} \widetilde{Y}_{z x}(\xi) \widetilde{e}_{z m}(\xi) \widetilde{e}_{x n}^{*}(\xi) d \xi \\
& K_{x x}=\int_{-\infty}^{\infty} \widetilde{Y}_{x x}(\xi) \widetilde{e}_{x m}(\xi) \widetilde{e}_{x n}^{*}(\xi) d \xi,
\end{aligned}
$$

$\mathrm{n}=1,2, \ldots, \ldots, \infty$ and asterisk denotes a complex conjugated quantity. In order that they have nontrivial solutions, the determinant of the matrix of equations must be zero. From this condition, known as the dispersion equation, the propagation constant $\gamma_{z}$ is determined at a chosen frequency. The frequency dependences of the phase constant and the attenuation constant are called dispersion characteristics. From the appearance of the dispersion characteristic, the behavior of a line can be predicted.

The crucial point is integration on the complex plane of the spectral variable $\xi$ in Eqs. (18) - (21). Integrands have poles and branch points. The same singularities are possessed by integrands of the backward Fourier transform when the field is searched for. Bound modes, modes leaking into the substrate and modes leaking into the space can propagate on the line. The respective propagation constant depends on the integration path. Integration along the real axis provides the phase constant of the bound mode. Integration along the real axis and round the poles carried out by the residual theorem provides the phase and the leakage constant of the mode leaking into the substrate. By analogy, when in addition the path crosses the branch cut around the branch point we again have the phase and leakage constant but power is taken away into the substrate and into the space.

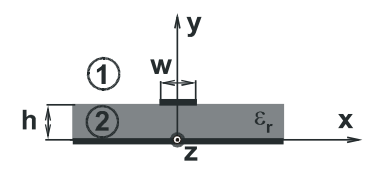

Fig. 2 Cross-section of a microstrip line.

The microstrip line, Fig. 2, is a dual structure to the slotline. Its analysis is the same as in the slotline case, but instead of the electric field in the slot there are longitudinal and transversal current density components on the strip. Eqs. (12) and (13) have the form

$$
\begin{aligned}
& \widetilde{E}_{z l}(\xi, h)=\widetilde{Z}_{z z}(\xi) \widetilde{J}_{z}(\xi, h)+\widetilde{Z}_{z x}(\xi) \widetilde{J}_{x}(\xi, h) \\
& \widetilde{E}_{x l}(\xi, h)=\widetilde{Z}_{x z}(\xi) \widetilde{J}_{z}(\xi, h)+\widetilde{Z}_{x x}(\xi) \widetilde{J}_{x}(\xi, h),
\end{aligned}
$$

where the series of basis functions $\tilde{j}_{z n}(\xi), \tilde{j}_{x n}(\xi)$ are

$$
\begin{aligned}
& \widetilde{J}_{z}(\xi, h)=\sum_{n=1}^{\infty} c_{z n} \widetilde{j}_{z n}(\xi) \\
& \widetilde{J}_{x}(\xi, h)=\sum_{n=1}^{\infty} c_{x n} \widetilde{j}_{x n}(\xi) .
\end{aligned}
$$

The basis functions have to fit as well as possible the electric field in the slot or the current density on the strip, in order to account for their behavior at the metal edge, and their Fourier transform must be available. We distinguish even and odd modes according to the symmetry of $E_{x}(x, h)$ or $J_{z}(x, h)$ with regard to the $y$ axis. In the past Chebyshev polynomials and circular functions combined with the Maxwell distribution, i. e., the edge-condition term, were used as basis functions. Both choices provide practically the same numerical results. Basis functions are nonzero only for $|x| \leq w / 2$. We work with circular functions and for even modes

$$
e_{z n}(x)=j_{x n}(x)=\frac{\sin [2 \pi n x / w]}{\sqrt{1-(2 x / w)^{2}}}
$$




$$
e_{x n}(x)=j_{z n}(x)=\frac{\cos [2(n-1) \pi x / w]}{\sqrt{1-(2 x / w)^{2}}},
$$

while for odd modes

$$
\begin{aligned}
& e_{z n}(x)=j_{x n}(x)=\frac{\cos [(2 n-1) \pi x / w]}{\sqrt{1-(2 x / w)^{2}}} \\
& e_{x n}(x)=j_{z n}(x)=\frac{\sin [(2 n-1) \pi x / w]}{\sqrt{1-(2 x / w)}^{2}} .
\end{aligned}
$$

A real planar line has a finite width of the substrate, as in Figs. 15 and 21. When its sidewalls are an extension of the ground, we use the Fourier transform

$$
\widetilde{\Phi}(\xi, y)=\frac{2}{b} \int_{-b / 2}^{b / 2} \Phi(x, y) e^{-j \xi_{n} x} d x
$$

where

$$
\xi_{n}= \begin{cases}2 n \pi / b & \text { for even mod es } \\ (2 n-1) / b & \text { for odd mod es }\end{cases}
$$

and $\mathrm{n}=0, \pm 1, \pm 2, \ldots \infty$. The calculation of the propagation constant goes on by analogy with the procedure given above. For brevity the basis functions can be found in [11]. The field components are determined by the backward Fourier transform

$$
\Phi(x, y)=\frac{1}{2} \sum_{n=-\infty}^{\infty} \widetilde{\Phi}\left(\xi_{n}, y\right) e^{j \xi_{n} x}
$$

Another important quantity, essential for the design of circuits, is the characteristic impedance of the dominant mode. Because it is a hybrid mode, the definition of the characteristic impedance is not unique. For the slotted lines we use the power-voltage definition

$$
Z=\frac{V^{2}}{2 P}
$$

and for strip lines we use the power-current definition

$$
Z=\frac{2 P}{I^{2}}
$$

$\mathrm{V}$ is the voltage across the slot

$$
V=\int_{-w / 2}^{w / 2} E_{x}(x) d x
$$

and I is the current flowing on the strip

$$
I=\int_{-w / 2}^{w / 2} J_{z}(x) d x
$$

$V$ and $I$ can be evaluated since integrands are defined by the series expansion and by the known amplitudes of the basis functions computed in the propagation constant search. $P$ is the time averaged power flow along the line and by Parseval's theorem it can be computed in the spectral domain.

\section{THEORETICAL ACHIEVEMENTS AND PRACTICAL CONSEQUENCES}

A survey of our achievements in recent years in investigating slotted planar transmission lines is presented in this section. The dispersion characteristics, field distribution and measured data will be accompanied by a brief commentary.

The stripline, the microstrip line, the coplanar waveguide and coplanar strips had already been investigated by several research groups round the world. This previous work therefore determined our initial orientation to the slotline and to its modifications.

It had been accepted for a long time that the excitation of the surface leaky mode demarcates the frequency band of pure bound mode propagation on a slotline. However, in addition to this known first leaky mode we revealed the occurrence of a second leaky mode, shown in Fig. 3, [10]. The field distribution computed by the CST Microwave Studio confirmed our theoretically predicted leaky modes. Fig. 4a shows $E_{y}$ of the 1 st and 2 nd leaky mode, while Fig. 4b demonstrates $H_{y}$ of the 2nd leaky mode and the decreasing amplitude of the field along the slot due to the leakage into the substrate.
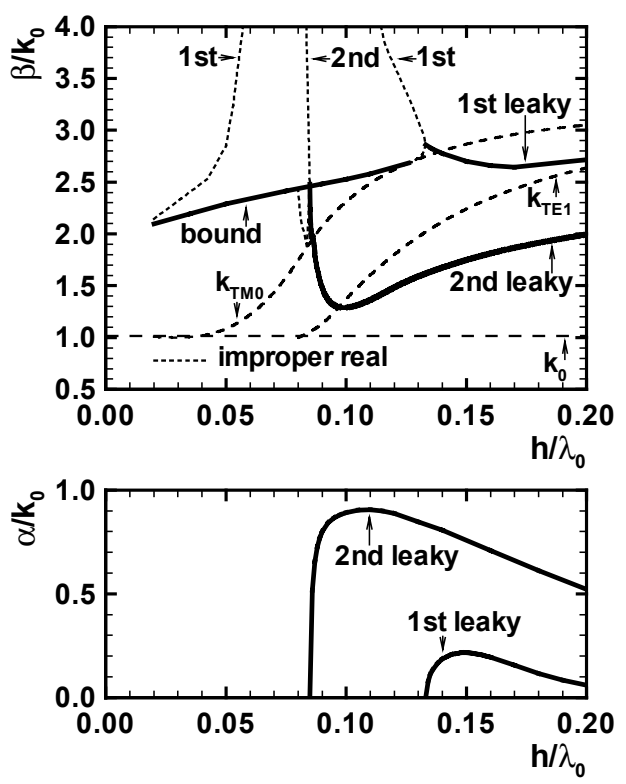

Fig. 3 The normalized phase and leakage constants for the slotline with $w=0.15 \mathrm{~mm}, h=0.635 \mathrm{~mm}, \varepsilon_{\mathrm{r}}=10.8$.

Three operation regimes can set in on a slotline in dependence on its proportions and the permittivity of the substrate, Fig. 5. Frequencies $f_{1}, f_{2}, f_{3}$ are the upper limits of pure bound mode propagation, since simultaneous propagation of the bound mode and leaky mode reduces the usable frequency band. On a slotline with a wider slotwidth the first leaky mode is predominant. The second leaky mode has greater significance on slotlines with narrower slotwidth 


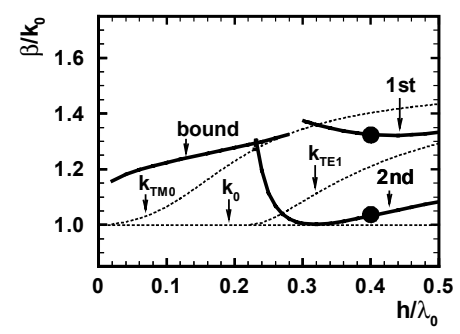

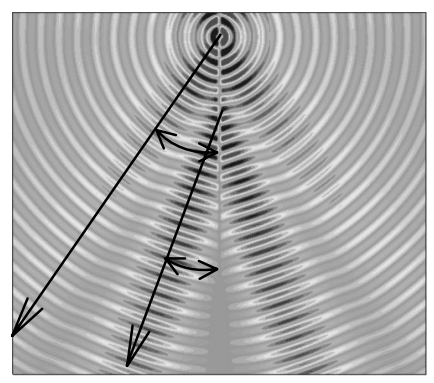

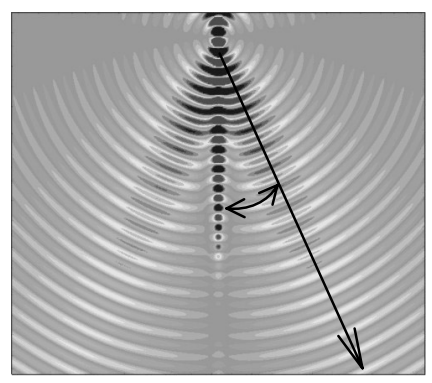

b
Fig. $4 E_{y}$ of the 1st and 2nd leaky mode in the substrate of a slotline with $w / h=0.4, \varepsilon_{\mathrm{r}}=2.25$ and $h / \lambda_{0}=0.4$, angles of leakage $\Theta_{l} \approx 19 \mathrm{deg}$ and $\Theta_{2} \approx 41 \mathrm{deg}$ (a), $H_{y}$ of the 2 nd leaky mode in the substrate of the same slotline, angle of leakage $\Theta_{2} \approx 30 \mathrm{deg}(\mathrm{b})$.

and made on a higher permittivity substrate. The essence of this thinking is shown in Figs. 6 and 7. Closed-form formulas of the normalized upper frequency cutoff of the dominant bound mode determined by the spectral gap $\left(h / \lambda_{c}\right)$, by the first leaky mode $\left(h / \lambda_{c 1}\right)$, and by the second leaky mode $\left(h / \lambda_{c 2}\right)$ are available for easy CAD of a slotline [10].
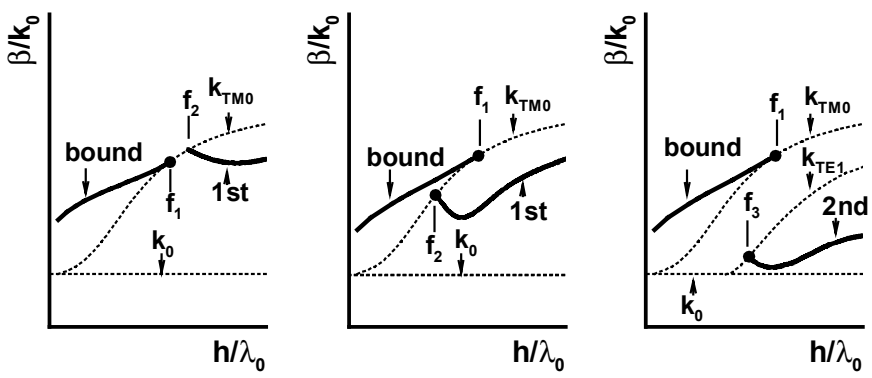

Fig. 5 Upper limits of bound mode propagation in three operation regimes.

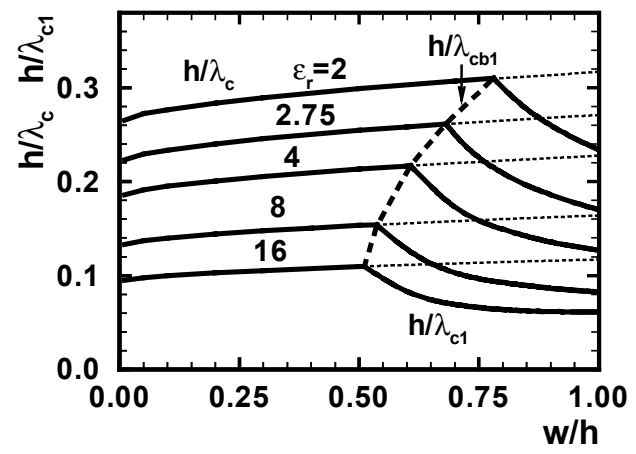

Fig. 6 The normalized upper frequency cutoff of the dominant bound mode determined by the spectral gap $\left(h / \lambda_{c}\right)$ and by simultaneous propagation of the first leaky mode $\left(h / \lambda_{c l}\right)$.

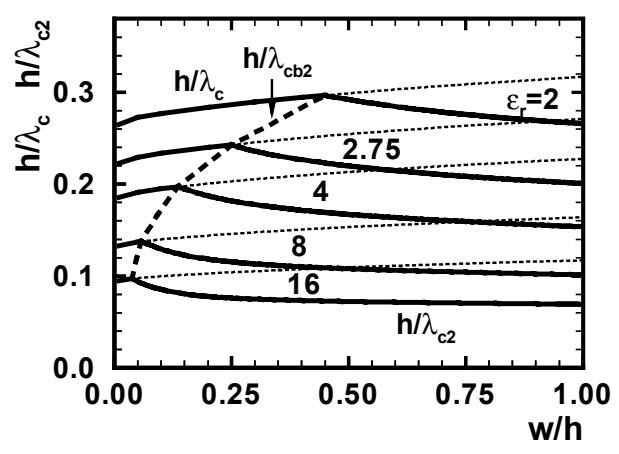

Fig. 7 The normalized upper frequency cutoff of the dominant bound mode determined by simultaneous propagation of the second leaky mode $\left(h / \lambda_{c 2}\right)$.

The slotline is sometimes located on the top of a cooling radiator or on the bottom of a shielding box. Consequently, we have a conductor-backed slotline, Fig. 8. In the past it was shown that only leaky modes are allowed on this line. We have revealed two new findings supplementing the previous thinking. These are: the existence of a dominant unbounded mode and double degeneration of the mode leaking into the substrate.

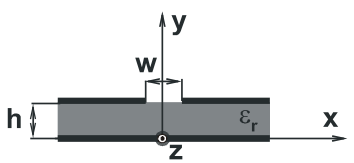

Fig. 8 Cross-section of a conductor-backed slotline.

It turned out that when the integration path on the complex plane $\xi$ is along the real axis on which the poles of the Green function are located, the propagation constant of that mode is real. The mode propagates unattenuatedly along the line and possesses a standing wave character in the lateral direction in the substrate, and has a zero cutoff. Such a field results from a TEM wave incident at an angle to the region below the slot, where the effective permittivity is lower than the permittivity of the substrate between conductive parallel plates. The total reflection springs up at the blurred boundary of these two permittivities. The field does not pass to the area on the opposite side of the slot, but is coupled through the slot to the air. The situation is illustrated in Fig. 9. However, the field excited by the real source is reflected from the rear and lateral walls of the substrate with finite size, and is incident under
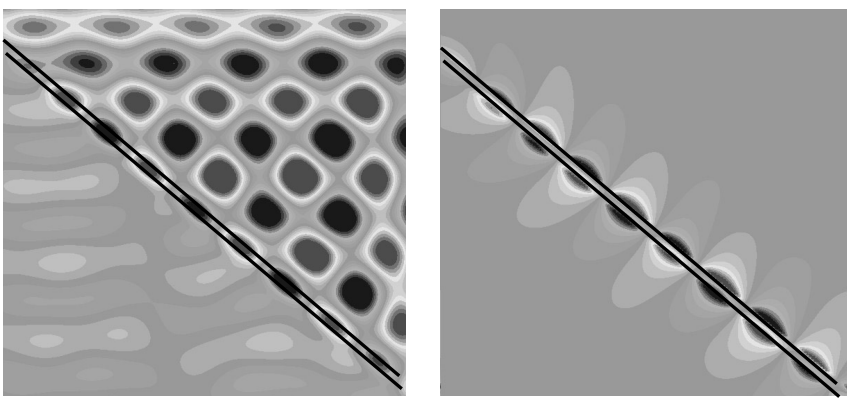

Fig. 9 Electric field component $E_{y}$ in, and perpendicular to, the substrate of the TEM mode, and coupled into air through the slot, when $w=h=6 \mathrm{~mm}, \varepsilon_{\mathrm{r}}=2.6$ and $f=5 \mathrm{GHz}$. 
various angles to the region below the slot. The reflections from the sidewalls substitute the absent source at infinity. The wavelength of the "semi-standing" wave varies slightly due to the different angles of incidence, as was confirmed by measurements, Fig. 10.

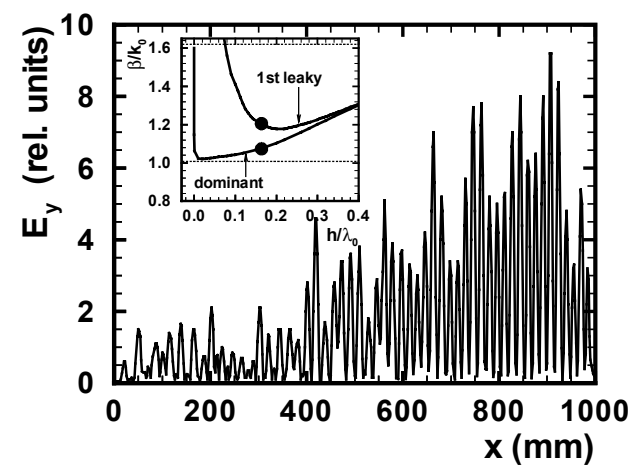

Fig. 10 Total $E_{y}$ component of the electric field in the transversal plane of the conductor-backed slotline when the dominant and 1st

leaky mode propagate, $w=h=6 \mathrm{~mm}, \varepsilon_{\mathrm{r}}=2.6$ and $f=5 \mathrm{GHz}$ or

$$
h / \lambda_{0}=0.16 \text {. }
$$

The dispersion equation has a multivalue solution. This feature has been manifested on the dispersion characteristics evolving with the slotwidth. When the slotwidth is greater than the substrate thickness the previous complex nonphysical solution merges with the complex physical solution. There are two "sub-modes" leaking at different angles into the substrate at one frequency. The situation is shown in Figs. 11 and 12.
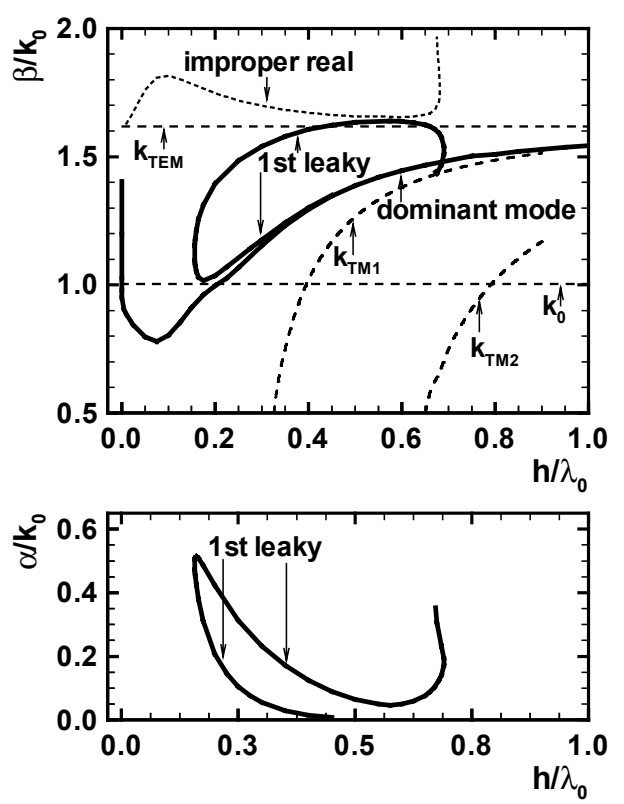

Fig. 11 The normalized dispersion characteristics of even modes on a conductor-backed slotline, when $w=10 \mathrm{~mm}, h=6 \mathrm{~mm}, \varepsilon_{\mathrm{r}}=2.6$.

A conductor-backed slotline is not suitable for signal transmission owing to the leakage and the presence of the not bound dominant mode. To eliminate this shortcoming the substrate is divided into two layers. The bottom layer has lower permittivity than the top layer. Now the dominant bound mode propagates from zero to the cutoff of the wave propagating in the partitioned dielectrics between parallel plates.

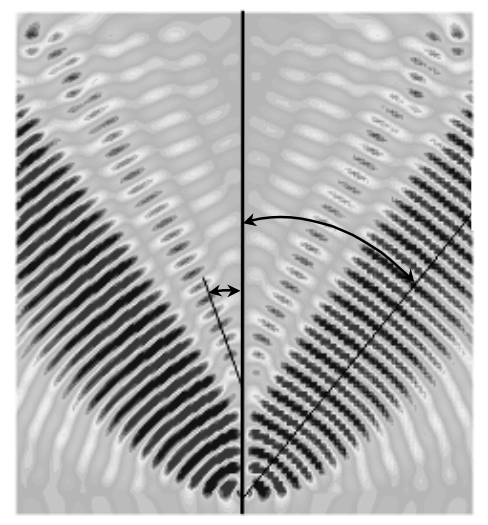

Fig. 12 Electric field component $E_{y}$ of the first even leaky modes in the substrate of a conductor-backed slotline with $w=10 \mathrm{~mm}, h=6 \mathrm{~mm}$, $\varepsilon_{\mathrm{r}}=2.6$ and $h / \lambda_{0}=0.3$.

Another more easily achievable solution is to modify the line by putting an additional dielectric on its top according to Fig. 13 and $\varepsilon_{2}>\varepsilon_{3}>\varepsilon_{1}$. The dispersion characteristics of the even bound and two leaky modes are shown in Fig. 14. The dominant mode is bound in all three dielectrics and propagates from zero frequency. We checked the excitation of that mode on the line with a plexiglass substrate and a glass slab on top, dots in Fig. 14. The first leaky mode leaks into the top slab while the second leaky mode leaks into the substrate and the top slab. The double degeneration of the mode leaking into the

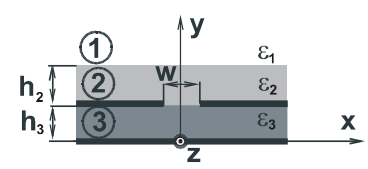

Fig. 13 Cross-section of a modified conductor-backed slotline.

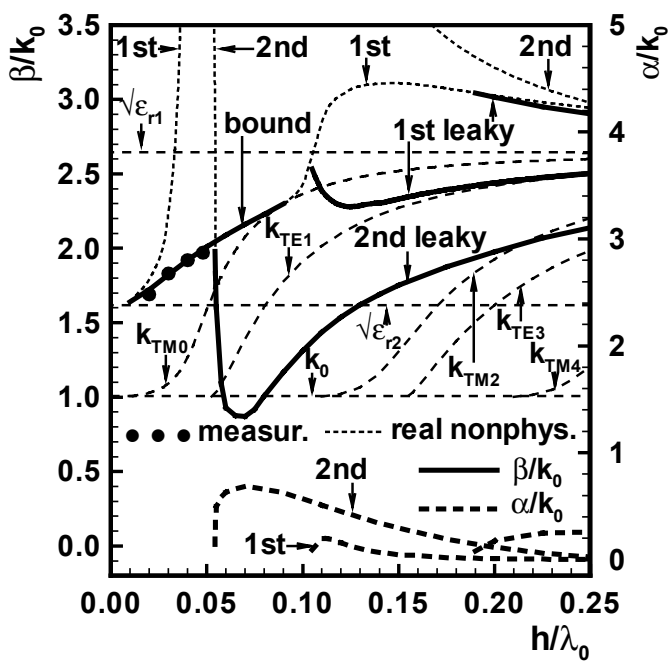

Fig. 14 Dispersion characteristics of the even modes on a modified conductor-backed slotline when $w=6 \mathrm{~mm}, h_{2}=12 \mathrm{~mm}, h_{3}=6 \mathrm{~mm}$,

$$
\varepsilon_{\mathrm{r} 2}=7, \varepsilon_{\mathrm{r} 3}=2.6 \text {. }
$$


upper slab was also discovered in this case. It can be a consequence of widening the slot or also lowering the height of the upper slab.

The safest way of preventing from surface and space leakage is total shielding of the line. Such a line does not suffer from losses of energy by radiation or cross talk, and has great electromagnetic resistance. In the case of a slotline or a conductor-backed slotline, this concept leads to the crosssection of the line shown in Fig. 15. This line is in fact a flat rectangular waveguide with a conductive partition in the $\mathrm{H}$ plane with two different dielectrics occupying the upper and lower portion of the cross-section, in other words, a finned waveguide.

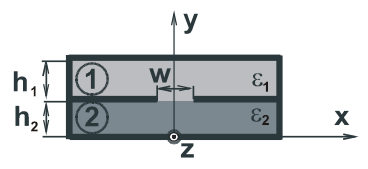

Fig. 15 Cross-section of a flat finned waveguide

Even and odd modes can exist on this line. Each has nonzero cutoff $f_{c}$ and the rise of the permittivity shifts them to lower frequencies. The frequency band of pure dominant mode propagation is only slightly influenced by the slot width and the permittivity. The smaller the waveguide width $b$, the greater is the change of the cut off with the change of the slot width. The cutoff strongly depends on the waveguide width. The greater $b$ is, the lower is $f_{c}$. There are two groups of modes. The field of the waveguide-type modes resembles the field of the TE modes in the rectangular waveguide perturbed now by the conductive partition in the $\mathrm{H}$ plane. The field of the slot-type modes resembles the field of the modes propagating along the conductor-backed slotline influenced now by the shielding. The increasing asymmetry in permittivities removes the distinction between the types of modes. The difference between the two types of modes is most distinct in the flat waveguide with $\varepsilon_{\mathrm{r} 1}=\varepsilon_{\mathrm{r} 2}=1$, as seen in Fig. 16 and 17.

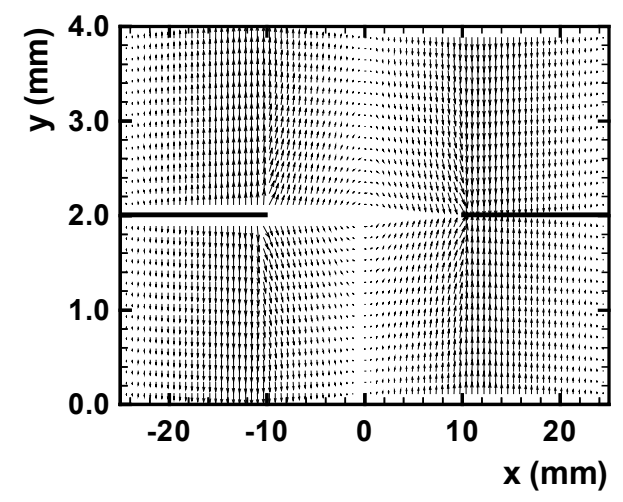

Fig. $16 E_{T}(x, y)$ of the even dominant slot-type mode in a finned waveguide when $h_{1}=h_{2}=2 \mathrm{~mm}, w=20 \mathrm{~mm}, b=50 \mathrm{~mm}, \varepsilon_{\mathrm{r} 1}=\varepsilon_{\mathrm{r} 2}=1, \mathrm{f}=6$ $\mathrm{GHz}$.

The real propagation constants of the revealed modes are shown in Figs. 18 and 19. They are compared with the data acquired by the CST Microwave Studio and by our measurements. Only the even dominant slot-type mode is significant for circuit applications. Its characteristic impedance changes easily by the ratio $h_{2} / h_{l}$, as shown in Fig. 20. The frequency dependence of the even and odd mode propagation constant is the same as in the rectangular waveguide. The corresponding cutoffs are available as the closed-form formula [11].

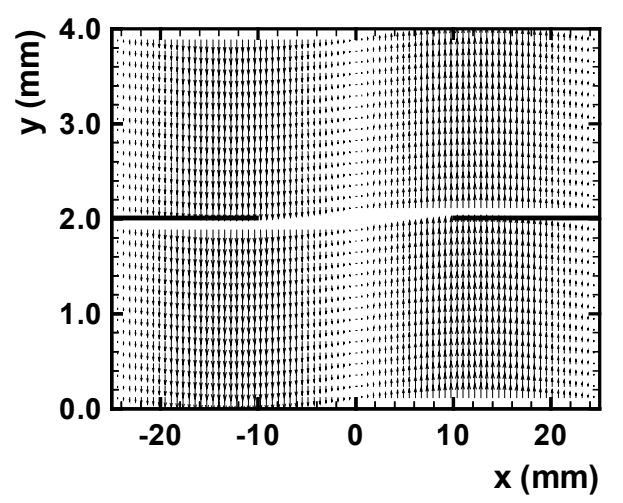

Fig. $17 E_{T}(x, y)$ of the first even waveguide-type mode in the finned waveguide specified in Fig. 16.

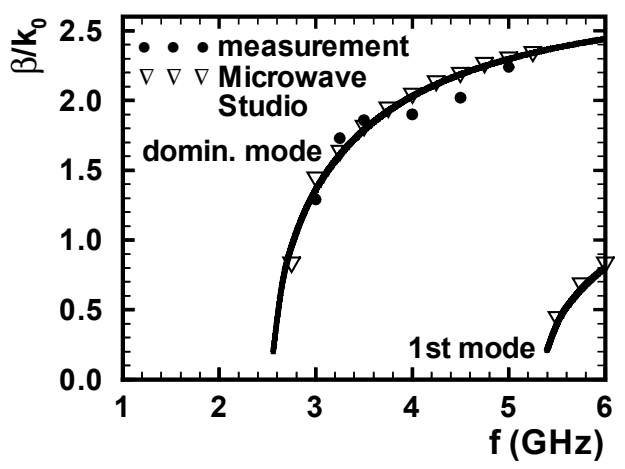

Fig. 18 Calculated and measured phase constant of the even dominant mode on a finned waveguide with $h_{1}=3 \mathrm{~mm}, h_{2}=5 \mathrm{~mm}$, $w=10 \mathrm{~mm}, b=30 \mathrm{~mm}, \varepsilon_{\mathrm{r} 1}=8, \varepsilon_{\mathrm{r} 2}=2.6$.

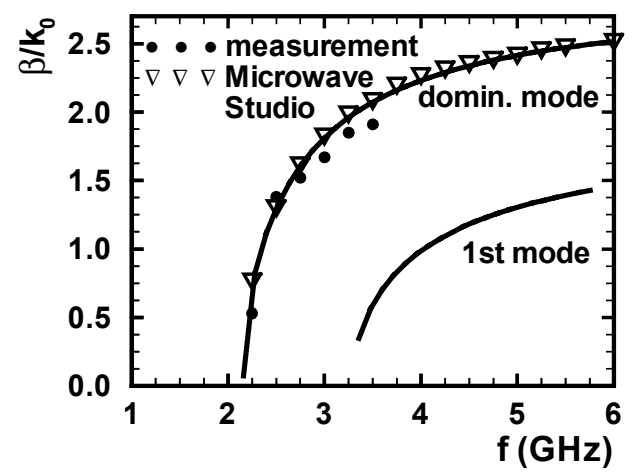

Fig. 19 Calculated and measured phase constant of the odd dominant mode on the finned waveguide specified in Fig. 18.

The motivation for investigating a flat waveguide with a longitudinal slot, Fig. 21, was the endeavour to design a flat, low profile antenna. This involves a conductor-backed slotline with a finitely wide substrate metallized also on its sidewalls. The analysis of this line is the alternative analysis of the 
standard rectangular waveguide with the slot cut in the middle of its wider wall.

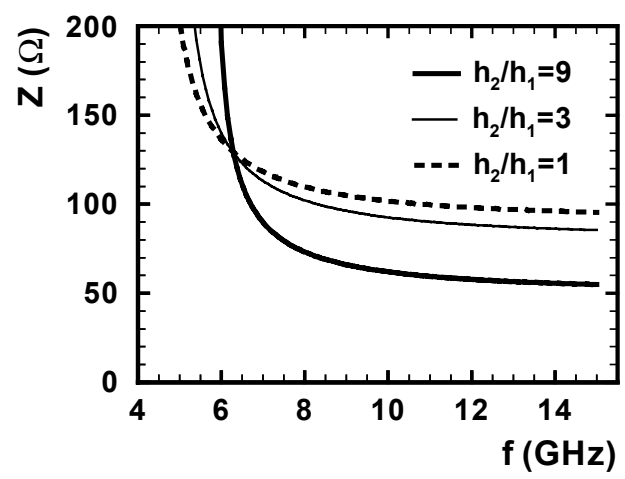

Fig. 20 Characteristic impedance of the even dominant mode of a finned waveguide when $h_{1}+h_{2}=10 \mathrm{~mm}, w=1 \mathrm{~mm}, b=23 \mathrm{~mm}$.

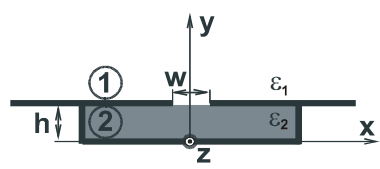

Fig. 21 Cross-section of a flat waveguide with a longitudinal slot.

Now the initial choice of the longitudinal component of the Hertz potentials is more advantageous. A specific aspect of the analysis is the matching of the fields in the spectral domain on the plane separating the boundless half-space and the bound volume of the substrate. This is equivalent to matching the Fourier integral and the Fourier series in the space domain. The relation between the Fourier transformed field components carried out according to Eqs. 2 and 30 is mediated by the sample function.

We have again identified even and odd modes on the guide. The dispersion characteristics of the first five modes are plotted in Fig. 22. These are the space leaky modes, and correspond to the modes of the rectangular waveguide perturbed by the longitudinal slot. The first space leaky mode is related to the dominant mode of the waveguide $\mathrm{TE}_{10}$. The second mode corresponds to the $\mathrm{TM}_{11}$ mode, and the field of the third mode resembles the field of the $\mathrm{TE}_{11}$ mode. The fourth space leaky mode is a modification of the $\mathrm{TM}_{12}$ mode, while the fifth has a link to the $\mathrm{TE}_{12}$ mode. Their phase constants trace the propagation constants of the modes in an unperturbed waveguide, dots in Fig. 22. Similarly, the attenuation constant, typical for evanescent modes below the cutoffs, coincides with the leakage constant, circles in Fig. 22. Accordingly, a waveguide with a narrow slot does not radiate since the leakage constant is very great below the cutoff. It also does not radiate above the cutoff, since the leakage constant is almost zero. The narrower the slot width, the closer together are the frequencies at which $\beta$ and $\alpha$ approach zero. The space leaky mode converts into a bound mode when $\beta=k_{0}$, as is shown in Fig. 23 drawn for even modes. When a dielectric slab with permittivity $\varepsilon_{\mathrm{r}}>1$ and thickness $h_{s}$ is placed on the top of the guide with $\varepsilon_{\mathrm{r} 1}=\varepsilon_{\mathrm{r} 2}=1$ in Fig. 21 we get the dispersion characteristics shown in Fig. 24. The initial space leaky mode converts into the bound mode, which leaks above the spectral gap into the slab.

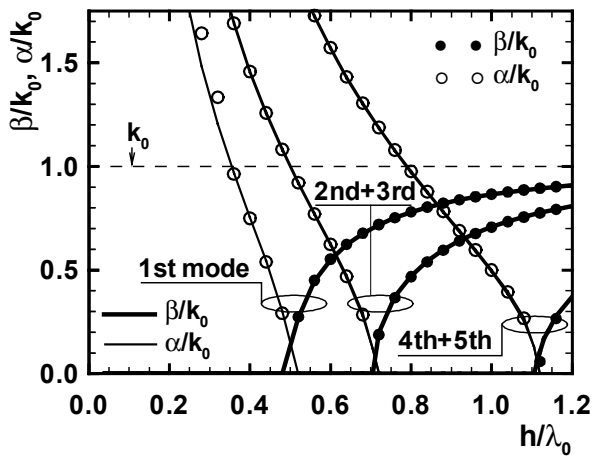

Fig. 22 Normalized dispersion characteristics of the odd space leaky modes on a squared slotted waveguide with $w=1 \mathrm{~mm}, h=b=10 \mathrm{~mm}$, $\varepsilon_{\mathrm{r} 1}=\varepsilon_{\mathrm{r} 2}=1$.

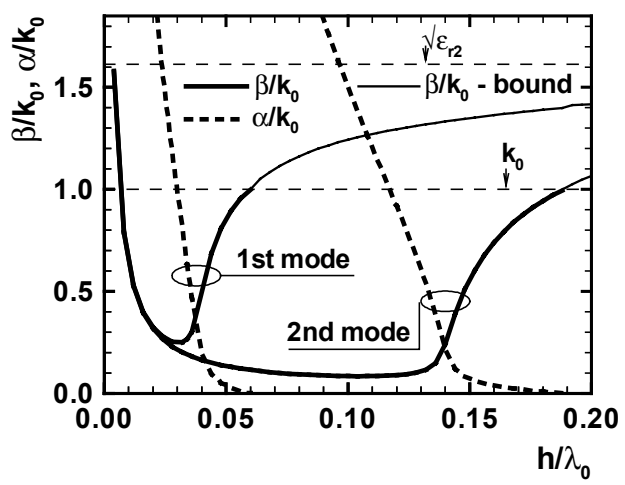

Fig. 23 Normalized dispersion characteristics of two even space leaky modes on a flat slotted waveguide when $w=2 \mathrm{~mm}, b=30 \mathrm{~mm}$, $h=6 \mathrm{~mm}, \varepsilon_{\mathrm{r} 2}=2.6, \varepsilon_{\mathrm{r} 1}=1$.

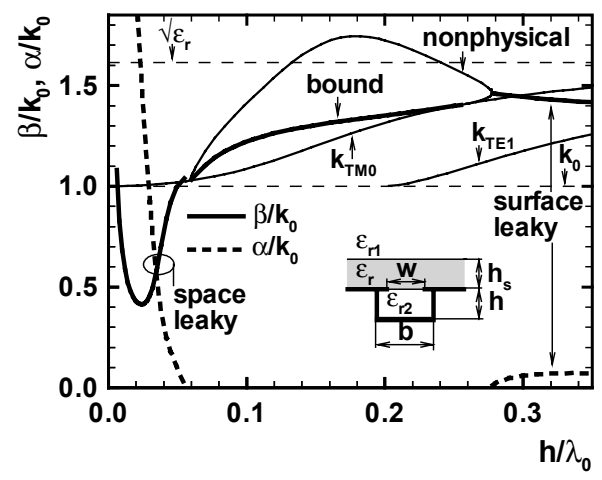

Fig. 24 Normalized dispersion characteristics of even modes on a flat slotted waveguide when $w=2 \mathrm{~mm}, b=30 \mathrm{~mm}, h=h_{s}=6 \mathrm{~mm}$,

$$
\varepsilon_{\mathrm{r} 2}=2.6, \varepsilon_{\mathrm{r} 1}=1 \text {. }
$$

In order to fill in the gap in code supply for researchers analyzing and designing planar transmission lines in wide frequency bands, we developed the APTL (Analysis of Planar Transmission Lines) package. It contains an analysis of 18 different planar transmission lines. The code provides the frequency dependent propagation constant of a selected mode, the characteristic impedance when it can be defined, the field distribution in the cross-section and the distribution of the transversal field component within a slot, or the current density component on the strip [13]. The code is open and analysis of the coplanar strips is currently being implemented. 
Based on our own experience, we can conclude that the design of open transmission lines is possible in the framework of the quasi-static concept only at relatively low frequencies. Insight into their behavior in a wide frequency band is provided only by the full wave approach. The APTL package is an example facilitating the work of the designer.

\section{ACKNOWLEDGEMENT}

Successive parts of this work have been supported by the Grant Agency of the Czech Republic under project 102/03/0449 "New circuit devices for communication technology".

\section{REFERENCES}

[1] R. K. Hoffmann, Integrierte Mikrowellenschaltungen, Berlin, Springer-Verlag, 1983.

[2] K. C. Gupta, R. Garg, I. J. Bahl, Microstrip Lines and Slotlines, Dedham, Artech House, 1979.

[3] B. Bhat, S. K. Koul, Analysis, Design and Applications of Fin Lines, Norwood, Artech House, 1987.

[4] B. C. Wadell, Transmission Line Design Handbook, Boston, Artech House, 1991.
[5] R. N. Simons, Coplanar Waveguide Circuits, Components, and Systems, New York, Wiley-Interscience, 2001.

[6] R. E. Collin, Field Theory of Guided Waves, 2nd ed., New York, IEEE Press, 1991.

[7] T. Itoh ed., Numerical Techniques for Microwave and Millimeter-Wave Passive Structures, New York, John Wiley \& Sons, 1989.

[8] T. Itoh, R. Mitra, "Dispersion Characteristics of Slotlines", Electron. Lett., vol. 7, no 13, pp. 364-365, 1971.

[9] T. Itoh, R. Mittra, "Spectral-domain Approach for Calculating Dispersion Characteristics of Microstrip Lines", IEEE Trans., Microwave Theory Tech., vol. 24, no. 7, pp. 496-498, 1973.

[10] J. Zehentner, J. Machač, M. Migliozzi, "Upper Cutoff Frequency of the Bound Wave and New Leaky Wave on the Slotline", IEEE Trans., Microwave Theory Tech., vol. 46, no. 4, pp. 378-386, 1998.

[11] J. Zehentner, J. Machac, J. Mrkvica, "Completely Shielded Conductor-Backed Slotline", submitted to IEEE Trans., Microwave Theory Tech., 2005.

[12] J. Zehentner, J. Mrkvica, J. Machac, "Flat Waveguide with Longitudinal Slot", accepted for 2005 IEEE MTT-S IMS, Long Beach, California, 2005.

[13] J. Mrkvica, Wave propagation on modified slotlines and coplanar lines, PhD Thesis (in Czech), Prague, CTU in Prague, 2004. 